\title{
OXYGEN AND SODIUM ABUNDANCES IN M13 (NGC 6205) GIANTS: LINKING GLOBULAR CLUSTER FORMATION SCENARIOS, DEEP MIXING, AND POST-RGB EVOLUTION
}

\author{
Christian I. Johnson ${ }^{1,3,4}$ and Catherine A. Pilachowski $^{2}$ \\ ${ }^{1}$ Department of Physics and Astronomy, UCLA, 430 Portola Plaza, Box 951547, Los Angeles, CA 90095-1547, USA; cijohnson@astro.ucla.edu \\ 2 Department of Astronomy, Indiana University, Swain West 319, 727 East Third Street, Bloomington, IN 47405-7105, USA; catyp@ astro.indiana.edu \\ Received 2012 May 2; accepted 2012 June 25; published 2012 July 17
}

\begin{abstract}
We present $\mathrm{O}, \mathrm{Na}$, and $\mathrm{Fe}$ abundances, as well as radial velocities, for 113 red giant branch (RGB) and asymptotic giant branch (AGB) stars in the globular cluster M13. The abundances and velocities are based on spectra obtained with the WIYN-Hydra spectrograph, and the observations range in luminosity from the horizontal branch (HB) to RGB tip. The results are examined in the context of recent globular cluster formation scenarios. We find that M13 exhibits many key characteristics that suggest its formation and chemical enrichment are well described by current models. Some of these observations include the central concentration of O-poor stars, the notable decrease in $[\mathrm{O} / \mathrm{Fe}]$ (but small increase in $[\mathrm{Na} / \mathrm{Fe}]$ ) with increasing luminosity that affects primarily the "extreme" population, the small fraction of stars with halo-like composition, and the paucity of O-poor AGB stars. In agreement with recent work, we conclude that the most O-poor M13 giants are likely He-enriched and that most (all?) O-poor RGB stars evolve to become extreme HB and AGB-manqué stars. In contrast, the "primordial" and "intermediate" population stars appear to experience standard HB and AGB evolution.
\end{abstract}

Key words: Galaxy: halo - globular clusters: general - globular clusters: individual (M13, NGC 6205) - stars: abundances - stars: Population II

Online-only material: color figures, machine-readable table

\section{INTRODUCTION}

For many years globular clusters (GCs) were viewed as prototypical simple stellar populations containing stars of a single age and chemical composition. However, a detailed examination of GC chemistry revealed large star-to-star abundance variations of the light elements from carbon through aluminum (e.g., see reviews by Kraft 1994; Gratton et al. 2004; 2012). While the anticorrelated behavior of carbon and nitrogen with increasing luminosity along the red giant branch (RGB) attributed to first dredge-up (e.g., Iben 1965) and "canonical extra mixing" (e.g., Denissenkov \& VandenBerg 2003), was observed in both cluster and field stars, a peculiar pattern of enhanced $\mathrm{N}, \mathrm{Na}$, and $\mathrm{Al}$ abundances coupled with depleted $\mathrm{O}$ and $\mathrm{Mg}$ seemed to be only found in some cluster stars. The simultaneous anticorrelation of $\mathrm{O}$ and $\mathrm{Mg}$ with $\mathrm{Na}$ and $\mathrm{Al}$ pointed to high-temperature protoncapture burning as the likely source. Unfortunately, it was not immediately clear if the processed material found in the photospheres of GC RGB stars was due to in situ mixing or pollution from a previous generation of more massive stars.

The comprehensive GC abundance survey by Carretta et al. (2009b, 2009c) verified that these light element "anomalies," in particular the $\mathrm{O}-\mathrm{Na}$ anticorrelation, are likely present in all Galactic GCs. Additionally, several authors have now shown that the large star-to-star light element abundance variations found on the RGB are also present along the lower RGB, subgiant branch (SGB), and main-sequence turnoff (e.g., Gratton et al.

\footnotetext{
3 Visiting Astronomer, Kitt Peak National Observatory, National Optical Astronomy Observatories, which is operated by the Association of Universities for Research in Astronomy, Inc. (AURA) under cooperative agreement with the National Science Foundation. The WIYN Observatory is a joint facility of the University of Wisconsin-Madison, Indiana University, Yale University, and the National Optical Astronomy Observatory.

4 National Science Foundation Astronomy and Astrophysics Postdoctoral Fellow.
}

2001; Cohen \& Meléndez 2005; Bragaglia et al. 2010). This observation indicates that the unique abundance patterns of GCs are the result of the cluster formation and subsequent evolution rather than in situ processing. Recent photometric observations have discovered that many (all?) GCs exhibit multiple evolutionary sequences in their color-magnitude diagrams (e.g., see reviews by Piotto 2009; Gratton et al. 2012). Since most clusters exhibit a $<0.1$ dex spread in $[\mathrm{Fe} / \mathrm{H}]^{5}$ (e.g., Carretta et al. 2009a), except for a few notable cases with significant $[\mathrm{Fe} / \mathrm{H}]$ dispersion, the multiple photometric sequences are believed to be driven by He abundance differences. While the source of $\mathrm{He}$, and subsequently the light element variations, is not known, plausible candidates include $\sim 5-9 M_{\odot}$ asymptotic giant branch (AGB) stars (e.g., Ventura \& D'Antona 2009, 2011), rapidly rotating massive main-sequence stars (e.g., Decressin et al. 2010), and massive binary stars (e.g., de Mink et al. 2009).

Although recent GC formation models incorporating winds from intermediate mass and massive stars mixed with pristine gas are able to reproduce many of the light element abundance trends unique to the cluster environment (e.g., Decressin et al. 2010; Valcarce \& Catelan 2011; D'Ercole et al. 2012), the very low oxygen abundances $([\mathrm{O} / \mathrm{Fe}]<-0.4)$ found in some $\mathrm{GC}$ RGB stars seem to require additional processing. While the old paradigm that the $\mathrm{O}-\mathrm{Na}$ anticorrelation is entirely driven by in situ deep mixing in cluster RGB stars is clearly incorrect, the discovery that many GC stars are also He-rich has an important consequence for resurrecting a modified deep mixing scenario. D'Antona \& Ventura (2007) showed that it is possible for a metal-poor star that is both He-rich and initially moderately O-poor $([\mathrm{O} / \mathrm{Fe}] \sim-0.2)$ and Na-rich to further deplete oxygen down to $[\mathrm{O} / \mathrm{Fe}] \sim-1$, without a significant change in the $[\mathrm{Na} / \mathrm{Fe}]$ ratio.

\footnotetext{
$[\mathrm{A} / \mathrm{B}] \equiv \log \left(N_{\mathrm{A}} / N_{\mathrm{B}}\right)_{\mathrm{star}}-\log \left(N_{\mathrm{A}} / N_{\mathrm{B}}\right)_{\odot}$ for elements $\mathrm{A}$ and $\mathrm{B}$.
} 


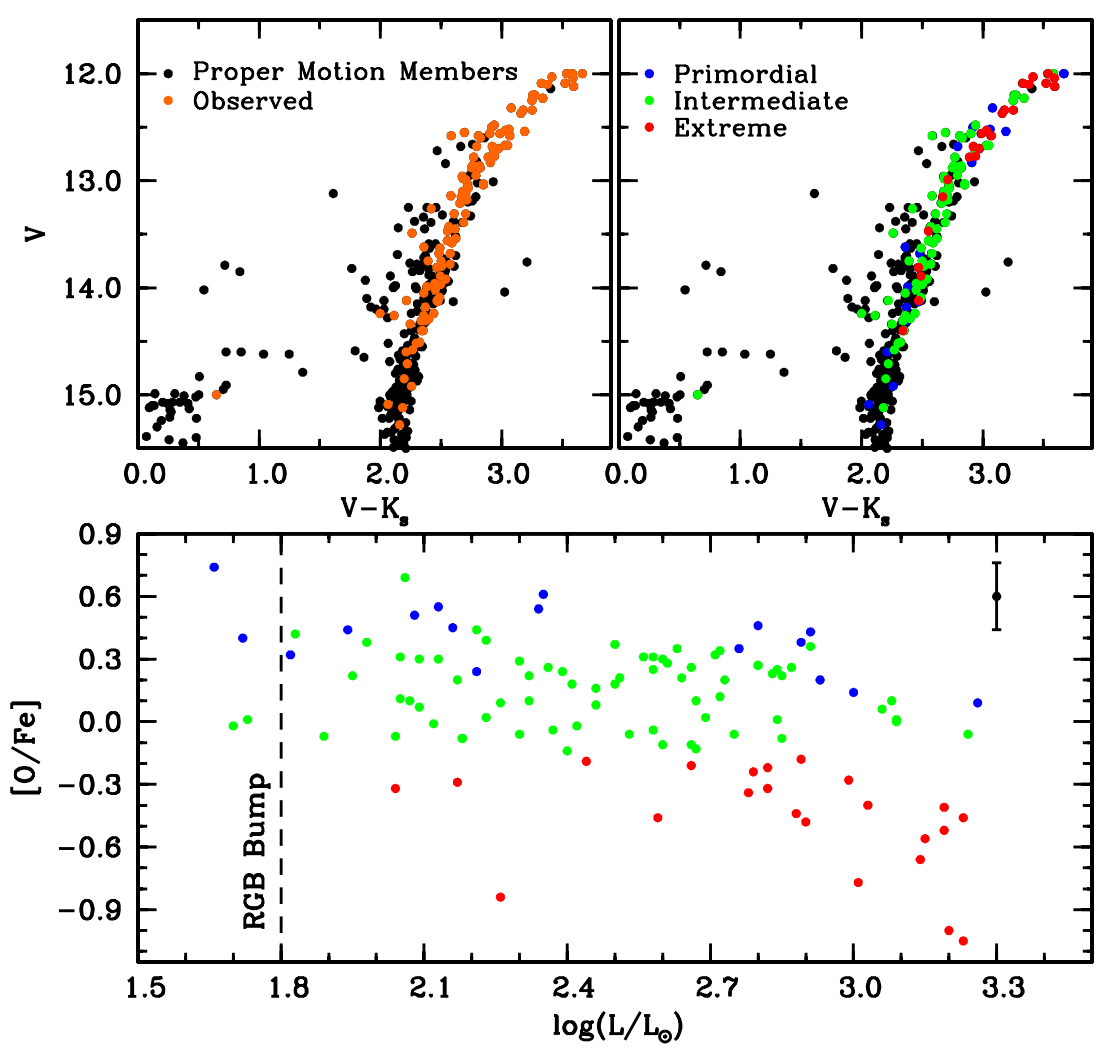

Figure 1. Top left panel shows a $V$ vs. $V-K_{\mathrm{S}}$ color-magnitude diagram indicating the stars observed for this program. The top right panel shows the same stars differentiated by chemical composition (see also Section 3). The bottom panel plots $[\mathrm{O} / \mathrm{Fe}] \mathrm{vs} . \log \left(L / L_{\odot}\right)$. The different color symbols have the same meaning as in the top right panel.

(A color version of this figure is available in the online journal.)

In light of this, M13 is a particularly illuminating case. It has long been known that M13 hosts some of the most O-poor and $\mathrm{Na} / \mathrm{Al}$-rich RGB stars of any cluster, and that these stars appear to be found preferentially near the RGB-tip (e.g., Kraft et al. 1992, 1997; Pilachowski et al. 1996; Cavallo \& Nagar 2000; Sneden et al. 2004; Cohen \& Meléndez 2005; Johnson et al. 2005). However, the sample size of stars for which $[\mathrm{O} / \mathrm{Fe}]$ has been determined is $\sim 5$ times less than that for which $[\mathrm{Na} / \mathrm{Fe}]$ and $[\mathrm{Al} / \mathrm{Fe}]$ have been measured. Since the $[\mathrm{O} / \mathrm{Fe}]$ ratio may be the most sensitive indicator of deep mixing (D'Antona $\&$ Ventura 2007), in this work we have measured [O/Fe] (and $[\mathrm{Na} / \mathrm{Fe}]$ ) abundances for $>100 \mathrm{RGB}$ and AGB stars ranging in luminosity from the RGB bump to the RGB tip. We now use this extended sample to examine how M13's extreme $\mathrm{O}-\mathrm{Na}$ anticorrelation extension fits into the modern picture of GC formation and evolution.

\section{OBSERVATIONS, DATA REDUCTION, AND ANALYSIS}

All observations for this project were obtained on 2011 May 19-20 using the WIYN $3.5 \mathrm{~m}$ telescope instrumented with the Hydra multifiber positioner and bench spectrograph. A single spectrograph setup, with wavelength coverage ranging from 6050 from $6350 \AA$, was used to obtain high signal-tonoise ratio $(\mathrm{S} / \mathrm{N}>100)$, moderate resolution $(R \approx 18,000)$ spectra of 113 RGB and AGB stars. A color-magnitude diagram illustrating the evolutionary state of the observed stars is shown in Figure 1. All coordinates, optical photometry, and membership probabilities were taken from Cudworth \& Monet (1979). Infrared photometry was taken from the Two Micron
All Sky Survey database (Skrutskie et al. 2006). To ensure membership, we only observed targets with $P>70 \%$.

The data reduction and analysis closely follow the techniques outlined in Johnson et al. (2005) and Johnson \& Pilachowski (2010). To briefly summarize, effective temperature $\left(T_{\text {eff }}\right)$ and surface gravity $(\log (g))$ were set for each star using dereddened $V$ and $K_{\mathrm{S}}$ photometry. We initially assumed $[\mathrm{Fe} / \mathrm{H}]=-1.50$ and a microturbulence (vt) value of $2 \mathrm{~km} \mathrm{~s}^{-1}$ and interpolated within the $\alpha$-enhanced, AODFNEW grid of ATLAS9 model atmospheres (Castelli et al. 1997). The final model metallicity was set as the average $[\mathrm{Fe} / \mathrm{H}]$ derived from $\mathrm{Fe}$ I and $\mathrm{Fe}$ II lines, and vt was set by removing any trend in $\mathrm{Fe} \mathrm{I}$ abundance versus line strength.

Abundances for $\mathrm{Fe} \mathrm{I}$ and $\mathrm{Fe}$ II were derived via equivalent width measurements while $\mathrm{O}$ and $\mathrm{Na}$ abundances were determined through spectrum synthesis. All abundances were calculated using the 2010 version of the LTE line analysis code MOOG (Sneden 1973). The line list was the same as that used in Johnson \& Pilachowski (2010). A summary of all derived model atmosphere parameters, coordinates, abundances, and radial velocities is provided in Table 1.

\section{BASIC RESULTS}

Despite exhibiting large light element abundance variations, M13 has always been characterized by a single metallicity $([\mathrm{Fe} / \mathrm{H}] \sim-1.5)$. We find in agreement with past large sample studies (e.g., Pilachowski et al. 1996; Sneden et al. 2004; Cohen \& Meléndez 2005) that M13 is moderately metal-poor and exhibits small star-to-star variation in $[\mathrm{Fe} / \mathrm{H}]$. In particular, we find $\langle[\mathrm{Fe} / \mathrm{H}]\rangle=-1.57(\sigma=0.07)$, with an average agreement 

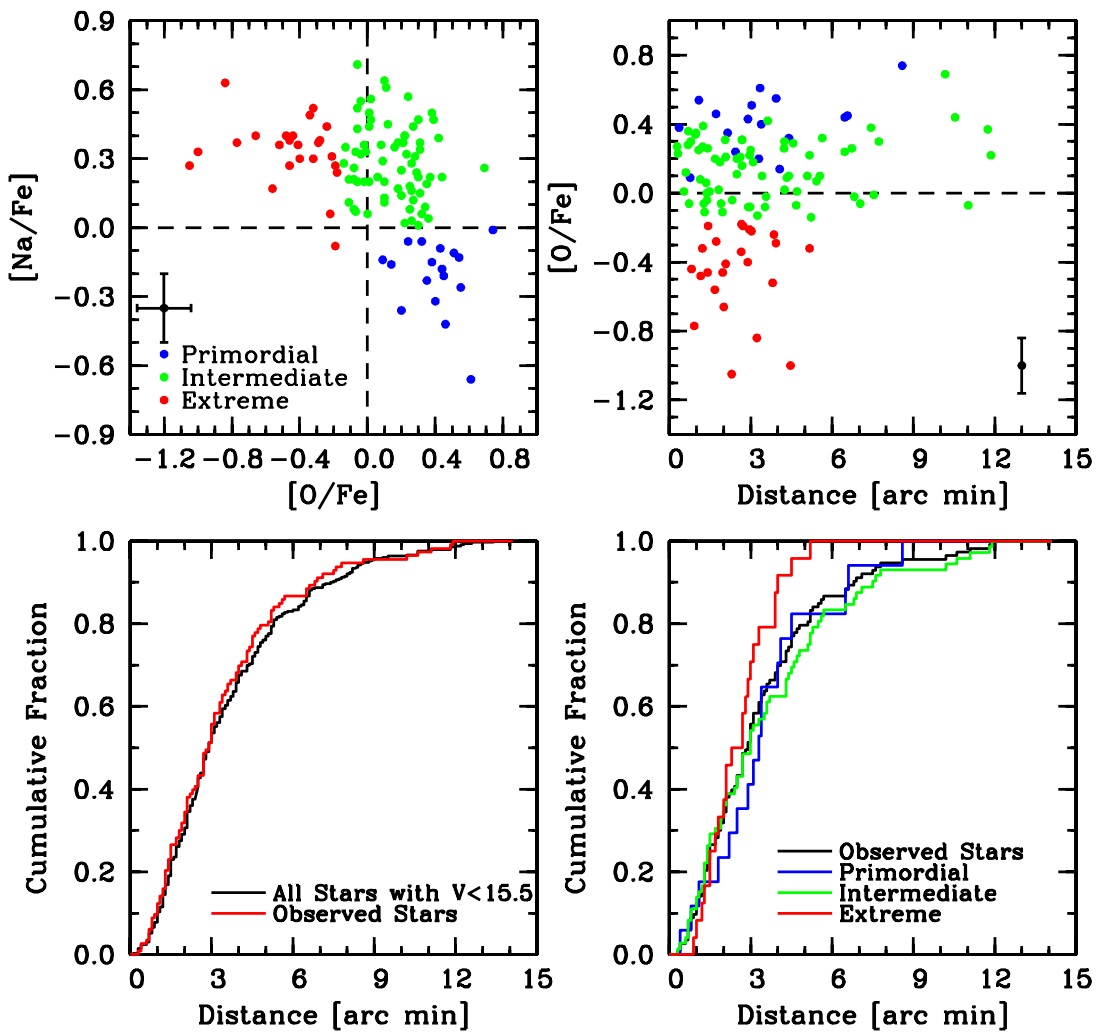

Figure 2. Top left panel plots $[\mathrm{Na} / \mathrm{Fe}]$ vs. $[\mathrm{O} / \mathrm{Fe}]$, and the top right panel shows $[\mathrm{O} / \mathrm{Fe}]$ vs. distance from the cluster center. The bottom left panel illustrates the cumulative fraction as a function of radial distance for all proper motion members (solid black line) and our observed distribution (solid red line). The bottom right panel compares the cumulative distribution of our observations based on composition.

(A color version of this figure is available in the online journal.)

Table 1

Basic Data and Results

\begin{tabular}{|c|c|c|c|c|c|c|c|c|c|c|c|c|c|c|c|}
\hline Star Name ${ }^{\mathrm{a}}$ & $\begin{array}{c}\text { Alt. } \\
\text { Name }\end{array}$ & $\begin{array}{l}\text { R.A. } \\
\text { J2000 }\end{array}$ & $\begin{array}{l}\text { Decl. } \\
\text { J2000 }\end{array}$ & V & $K_{\mathrm{S}}$ & $\log \left(L / L_{\odot}\right)$ & $\begin{array}{l}T_{\text {eff }} \\
(\mathrm{K})\end{array}$ & $\begin{array}{c}\log (g) \\
(\operatorname{cgs})\end{array}$ & $\begin{array}{c}{[\mathrm{Fe} / \mathrm{H}]} \\
\text { avg. }\end{array}$ & $\begin{array}{c}\mathrm{vt} \\
\left(\mathrm{km} \mathrm{s}^{-1}\right)\end{array}$ & {$[\mathrm{Fe} \mathrm{I} / \mathrm{H}]$} & {$[\mathrm{Fe}$ II $/ \mathrm{H}]$} & {$[\mathrm{O} / \mathrm{Fe}]$} & {$[\mathrm{Na} / \mathrm{Fe}]$} & $\begin{array}{c}\mathrm{RV} \\
\left(\mathrm{km} \mathrm{s}^{-1}\right)\end{array}$ \\
\hline 324 & V11 & 250.402711 & .443214 & 12.00 & 8.465 & 3.23 & 3955 & 0 & 1.50 & 2.50 & -1.67 & -1.32 & -0.46 & 27 & -242.7 \\
\hline L 598 & $\ldots$ & 250.424834 & 36.447735 & 12.00 & 8.335 & 3.26 & 3895 & 0.40 & -1.44 & 2.20 & - & - & +0.09 & 14 & -257.6 \\
\hline L 194 & II -90 & 250.383343 & 36.474979 & 12.03 & 8.616 & 3.19 & 4015 & 0.50 & -1.49 & 2.30 & -1.53 & -1.45 & -0.41 & +0.36 & -239.3 \\
\hline L 973 & $\mathrm{I}-48$ & 250.462198 & 36.481819 & 12.04 & 8.452 & 3.23 & 3930 & 0.45 & -1.50 & 2.35 & -1.68 & -1.32 & -1.05 & +0.27 & -249.5 \\
\hline
\end{tabular}

Notes.

${ }^{a}$ Designations from Ludendorf (1905) and Kadla (1966).

(This table is available in its entirety in a machine-readable form in the online journal. A portion is shown here for guidance regarding its form and content.)

between $[\mathrm{Fe} / \mathrm{H}]$ derived by $\mathrm{Fe}_{\mathrm{I}}$ and $\mathrm{Fe}$ II (in the sense $[\mathrm{Fe} \mathrm{I} / \mathrm{H}]-[\mathrm{Fe} \mathrm{II} / \mathrm{H}])$ of $-0.04(\sigma=0.15)$. The Fe I abundances are based on an average of 27 lines $(\sigma=4)$, with a typical lineto-line dispersion of $0.13 \mathrm{dex}(\sigma=0.02)$. In contrast, the Fe II abundances are based on 1-3 lines, with an average line-to-line dispersion of 0.11 dex $(\sigma=0.07)$. While the agreement between $[\mathrm{Fe} \mathrm{I} / \mathrm{H}]$ and $[\mathrm{Fe} \mathrm{II} / \mathrm{H}]$ is good for most stars, Table 1 shows that there is some disparity for the coolest, most luminous giants. This is likely due to a combination of mass loss, variability (if $V \lesssim 12.5$; Kopacki et al. 2003), model atmosphere deficiencies, and non-LTE (NLTE) effects.

In agreement with past work (e.g., Pilachowski et al. 1996; Kraft et al. 1997; Cavallo \& Nagar 2000; Sneden et al. 2004; Cohen \& Meléndez 2005; Johnson et al. 2005), we find large star-to-star abundance variations for both $[\mathrm{O} / \mathrm{Fe}]$ and $[\mathrm{Na} / \mathrm{Fe}]$ and reproduce the well-known $\mathrm{O}-\mathrm{Na}$ anticorrelation (see Figure 2). The $[\mathrm{O} / \mathrm{Fe}]^{6}$ ratio ranges from -1.05 to +0.74 , with an average $[\mathrm{O} / \mathrm{Fe}]=+0.06(\sigma=0.34)$. Similarly, $[\mathrm{Na} / \mathrm{Fe}]$ ranges from -0.66 to +0.71 , with an average of $[\mathrm{Na} / \mathrm{Fe}]=+0.23(\sigma=0.24)$. For $\mathrm{Na}$, the average measurement error is $0.08 \operatorname{dex}(\sigma=0.07)$. While the $\mathrm{O}$ abundance is derived solely from the $6300 \AA$ [O I] line, the typical synthesis fitting uncertainty is $\lesssim 0.1$ dex.

We did not apply any NLTE corrections to the $[\mathrm{Na} / \mathrm{Fe}]$ abundances. Although departures from LTE are expected for cool, metal-poor giants, the magnitude of the corrections likely does not exceed $\sim 0.1$ dex for the $6154 / 6160 \AA \mathrm{Na}$ I lines (e.g., Lind et al. 2011). The results presented here indicate a correlation between luminosity and the $\mathrm{O}-\mathrm{Na}$ anticorrelation, and it is important to ensure that this result is not purely a

\footnotetext{
6 Note that we measured $[\mathrm{O} / \mathrm{Fe}]$ relative to $[\mathrm{Fe} / \mathrm{H}]_{\text {avg. }}$ rather than $[\mathrm{Fe} \mathrm{II} / \mathrm{H}]$.
} 


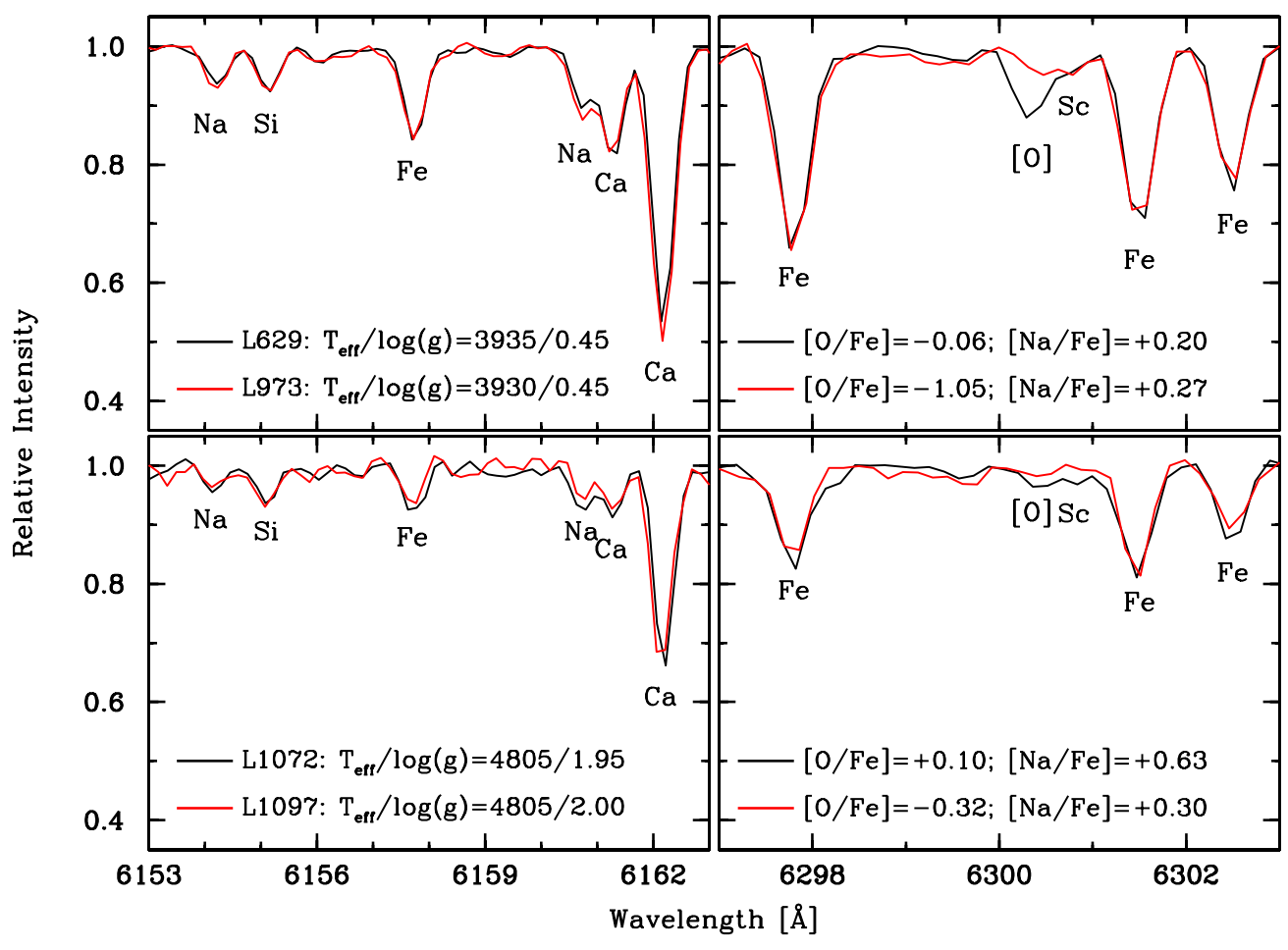

Figure 3. Sample spectra for two sets of stars with similar $T_{\text {eff }} / \log (\mathrm{g})$ but different $[\mathrm{O} / \mathrm{Fe}]$ and $[\mathrm{Na} / \mathrm{Fe}]$ abundances.

(A color version of this figure is available in the online journal.)

consequence of NLTE effects or model atmosphere deficiencies. While it is difficult to completely rule out these effects, we note that (1) abundance analyses of evolved RGB stars in the similar metallicity GC M3 do not find a correlation between $\mathrm{O} / \mathrm{Na}$ abundance and luminosity (e.g., Sneden et al. 2004; Cohen \& Meléndez 2005) and (2) as can be seen in Figure 3 there is a clear variation in $\mathrm{O} / \mathrm{Na}$ line strength among stars of similar luminosity.

Following the typical naming scheme for GC sub-populations (e.g., Carretta et al. 2009c), in Figures 1 and 2 we differentiate M13 stars into the "primordial," "intermediate," and "extreme" populations based on their $[\mathrm{O} / \mathrm{Fe}]$ and $[\mathrm{Na} / \mathrm{Fe}]$ abundances. ${ }^{7} \mathrm{We}$ find that the primordial, intermediate, and extreme populations constitute $15 \%, 63 \%$, and $22 \%$ of our sample, respectively, which is typical for Galactic GCs (e.g., Carretta et al. 2009c). Interestingly, as can be seen in Figures 1 and 2, the extreme population seems to differentiate itself by consisting of stars predominantly near the RGB tip, a result noted in many past studies (e.g., Kraft et al. 1997), and is the most centrally concentrated. Additionally, we note that none of the AGB stars in our sample are particularly O-poor (see also Pilachowski et al. 1996). We discuss the implications of these observations further in Section 4.

In addition to determining abundance ratios, we also measured radial velocities for all stars using the IRAF fxcor routine. We find an average heliocentric radial velocity (RV) of $-244.7 \mathrm{~km} \mathrm{~s}^{-1}(\sigma=6.1)$, which is in good agreement with past studies (e.g., Lupton et al. 1987). The average measurement error is $\sim 0.2 \mathrm{~km} \mathrm{~s}^{-1}$. The small star-to-star velocity dispersion indicates that all of our observed targets are likely cluster members. Interestingly, the extreme population exhibits an average

\footnotetext{
7 Note that our definitions are slightly different than those used in previous studies. Here we designate extreme stars by $[\mathrm{O} / \mathrm{Fe}]<-0.15$, primordial stars by $[\mathrm{Na} / \mathrm{Fe}]<+0.00$, and the remainder as intermediate stars.
}

$\mathrm{RV}$ that is $\sim 2 \mathrm{~km} \mathrm{~s}^{-1}$ larger than the primordial and intermediate stars, which have identical average RVs. However, this may be due to the fact that most extreme population stars are near the RGB tip and therefore likely to be variables (e.g., Kopacki et al. 2003).

\section{DISCUSSION AND CONCLUSIONS}

As mentioned in Section 1, recent models predict that GCs likely form in (at least) two distinct episodes. In this scenario, the first star formation event produces stars with halo-like composition (the primordial population), and then the $\gtrsim 5 M_{\odot}$ progeny of the first generation pollute the cluster with material heavily processed by high-temperature protoncapture burning, including newly synthesized He. This new material may be funneled to the cluster core (e.g., D'Ercole et al. 2008) where the second generation stars (intermediate and extreme populations) form; however, it appears that some dilution with pristine gas is required to reproduce the observed light element abundance trends (e.g., Prantzos et al. 2007; D'Ercole et al. 2011). Some implications of this scenario are that (1) the primordial population is preferentially stripped relative to the second generation stars, (2) the second generation stars may be significantly He-enhanced and more centrally concentrated, and (3) the extra $\mathrm{He}$, in addition to producing multiple evolutionary sequences in cluster color-magnitude diagrams, may cause some stars to experience in situ deep mixing above the RGB bump and/or cause the most He-rich stars to become RGB-manqué, AGB-manqué, or extreme blue horizontal branch (HB) stars. As we discuss below, M13 appears to exhibit many of these characteristics. ${ }^{8}$

\footnotetext{
8 Interestingly, multiple sequences in M13 color-magnitude diagrams have yet to be found (see Sandquist et al. 2010 for a recent update.)
} 


\subsection{Supporting Observations of Globular Cluster Formation Models}

We noted in Section 3 that the primordial population constitutes a considerably smaller fraction of stars in M13 (15\%) than the intermediate and extreme populations. This is consistent with the cluster formation scenario mentioned above, where a significant percentage (up to $\sim 90 \%$ ) of first generation, but not second generation, stars are lost early in the formation process. Although our estimate is somewhat lower than the $34 \%$ primordial fraction determined by Carretta et al. (2009c; their Table 5), we note that there is typically no clear separation between the primordial and intermediate populations. However, the dominance of the intermediate population in M13 strongly suggests that its formation and chemical enrichment followed the same path as other halo GCs.

Similarly, we show in Figure 2 that the extreme population appears to be marginally more centrally concentrated than the primordial and intermediate stars. This is supported by the results of two-sided KS tests, which indicate that the primordial and intermediate populations trace the same radial distribution $(\mathrm{KS}-\mathrm{prob}=0.9018)$ but the extreme population is different than both $\left(\mathrm{KS}-\right.$ prob $_{\mathrm{P}, \mathrm{E}}=0.1603$; KS-prob $\left.{ }_{\mathrm{I}, \mathrm{E}}=0.0935\right)$. Although the statistical significance is marginal, we note that similar results have been found in a few other clusters where the central concentration of extreme stars is supported by independent observations of radial changes in the color-magnitude diagram (e.g., Carretta et al. 2010; Lardo et al. 2011). While the dynamical evolution of a GC is expected to smear out the radial profile and uniformly mix the various populations after a Hubble time (e.g., Decressin et al. 2010; but see also Bekki 2010), the fact that M13 and other clusters still show a semblance of the extreme stars being centrally concentrated is evidence in support of current cluster formation models. In this light, $\omega$ Cen is a particularly illustrative example. Since the core relaxation time is similar to the cluster age, $\omega$ Cen likely preserves early formation history clues. In fact, Johnson \& Pilachowski (2010) and Gratton et al. (2011) find a clear composition dependence on radial location, with the extreme stars being the most centrally concentrated and the primordial stars the least.

\subsection{Connecting to the New Deep Mixing Model}

Although we now know that the historical argument relating in situ deep mixing and the $\mathrm{O}-\mathrm{Na}$ anticorrelation is incorrect, a modified version has recently been resurrected to explain cluster RGB stars with $[\mathrm{O} / \mathrm{Fe}] \lesssim-0.4$ (e.g., D'Antona \& Ventura 2007). As mentioned in Section 1, predicted yields from both $>5 M_{\odot}$ AGB and massive main-sequence stars generally fail to produce second generation stars with $[\mathrm{O} / \mathrm{Fe}] \lesssim-0.4$ and thus a secondary process is required.

Interestingly, our M13 observations (and those of past authors) appear to verify the predictions of the D'Antona \& Ventura (2007) model (see also Figure 1): (1) all of the known stars with $[\mathrm{O} / \mathrm{Fe}] \lesssim-0.4$ are located well above the RGB bump, (2) in general there is a monotonic decrease in $[\mathrm{O} / \mathrm{Fe}]$ with increasing luminosity for the extreme population, and (3) at the highest luminosity there is a large difference in $\langle[\mathrm{O} / \mathrm{Fe}]\rangle$ between the extreme and intermediate populations but only $\sim 0.1$ dex increase in $\langle[\mathrm{Na} / \mathrm{Fe}]\rangle$ for the extreme stars. We believe that the requirements to induce deep mixing (enhanced $\mathrm{He}$ and initially low $[\mathrm{O} / \mathrm{Fe}]$ ) are also met for the extreme M13 giants.
Figure 1 shows that (with one exception) the lowest $[\mathrm{O} / \mathrm{Fe}]$ ratio found at $\log \left(L / L_{\odot}\right)<2.8$ is consistent at $[\mathrm{O} / \mathrm{Fe}] \sim-0.3$. Note that this is consistent with the Cohen \& Meléndez (2005) observations that do not find stars below the RGB bump with $[\mathrm{O} / \mathrm{Fe}]<-0.2$. This supports the idea that the low $[\mathrm{O} / \mathrm{Fe}]$ values found only in the brightest M13 RGB stars is an evolutionary effect and that significant O-depletion does not occur at low RGB luminosities. With regard to He-enhancement, we do not have direct $\mathrm{He}$ measurements for these stars but note that the most $\mathrm{Na} / \mathrm{Al}$-rich (and thus O-poor) stars in $\omega \mathrm{Cen}$ (Dupree et al. 2011) and NGC 2808 (Pasquini et al. 2011) have enhanced He. We also find ancillary evidence, similar to that found by Carretta et al. (2006) in NGC 2808, in support of $\mathrm{He}$-enrichment from the increase in $[\mathrm{Fe} / \mathrm{H}]$ from -1.58 in the intermediate population to -1.54 in the extreme population. ${ }^{9}$ However, we note that Sandquist et al. (2010) do not find significant evidence for He-enrichment in M13. On the other hand, if the O-poor stars are He-rich then the fact that deep mixing appears to be activated at a single luminosity $\left(\log \left(L / L_{\odot}\right) \sim\right.$ 2.8) may be evidence in support of the extreme stars having a small He spread. This is qualitatively in agreement with photometric studies that often find discrete populations in cluster color-magnitude diagrams rather than a spread (e.g., Piotto 2009).

\subsection{Composition and Post-RGB Evolution}

In the scenario described above, the most He-rich stars likely undergo deep mixing that has the observational effect of decreasing $[\mathrm{O} / \mathrm{Fe}]$; however, it also increases the envelope He abundance to as much as $Y=0.5$ (e.g., D'Antona \& Ventura 2007). Since He-enhancement may be strongly manifest in HB and AGB evolution, we can look at these stars for clues regarding He-enhancement and RGB evolution. One of the most notable features of Figure 1, which has been shown previously with $\mathrm{Na}$ abundances (e.g., Pilachowski et al. 1996), is the lack of extreme stars on the AGB. ${ }^{10}$ Since we find the extreme stars to constitute $\sim 20 \%$ of M13's total population, we should expect to find $\sim 2-3$ super O-poor AGB stars in our sample. Interestingly, we find that only the primordial and intermediate AGB stars are present in about the same proportion as on the RGB. Following similar results in other GCs (e.g., Norris et al. 1981; Campbell et al. 2010; Gratton et al. 2010), we conclude that in M13 only the primordial and intermediate populations undergo standard $\mathrm{HB}$ and AGB evolution.

What about the fate of the extreme population? M13 is known to contain a bimodal and extreme blue HB (e.g., see Sandquist et al. 2010 and references therein). Circumstantial evidence supports the idea that the "faint peak" population of HB stars, which have very high $T_{\text {eff }}$, was also once the most O-poor population on the RGB. In particular, the fraction of the faint peak relative to the total $\mathrm{HB}$ stars is about equal to the fraction of extreme to total RGB stars. The faint peak stars were also found by Sandquist et al. (2010) to be more centrally concentrated than the "intermediate" and "bright peak" populations. Furthermore, the Sandquist et al. (2010) data indicate that (1) the fraction of AGB-manque to total AGB stars is $\sim 23 \%$ and (2) the origin of the AGB-manqué stars is likely the bluest part of the HB.

\footnotetext{
9 We caution the reader on this point because the $[\mathrm{Fe} / \mathrm{H}]$ difference is small and several of the extreme population stars are known to be variable.

${ }^{10}$ With the present data we are unable to differentiate AGB and RGB stars near the RGB tip. However, we expect most, if not all, of the stars near the RGB tip to be first ascent giants because of the short evolutionary timescale of AGB stars.
} 
Additionally, Peterson et al. (1995) provide [O/Fe] abundances for cool HB stars in M13 and do not find any with $[\mathrm{O} / \mathrm{Fe}]<0$. All of these observations suggest that the extreme RGB stars evolve from the RGB to the bluest end of the $\mathrm{HB}$ and then become AGB-manqué stars.

\subsection{Final Thoughts}

The results presented here have allowed us to re-examine M13 in light of recent advances in our understanding of GC formation. M13 may be well explained by the new "standard" picture in which first generation stars with halo-like composition are preferentially lost early in the cluster evolution, and a second, more enriched population forms in the cluster center from gas processed and ejected by $>5 M_{\odot}$ first generation stars. For M13, this has the effect of instigating in situ deep mixing in the most He-rich giants and perhaps causing them to terminate their evolution before ascending the AGB. In fact, proper modeling of the warmest $\mathrm{HB}$ stars in clusters like M13 may require considering composition changes to the RGB envelope due to in situ mixing. However, two outstanding issues remain: (1) will precise photometry in the inner part of the cluster finally reveal multiple populations? and (2) are some M13 stars actually He-rich?

This material is based upon work supported by the National Science Foundation under award No. AST-1003201 to C.I.J. C.A.P. gratefully acknowledges support from the Daniel Kirkwood Research Fund at Indiana University.

\section{REFERENCES}

Bekki, K. 2010, ApJ, 724, L99

Bragaglia, A., Carretta, E., Gratton, R. G., et al. 2010, ApJ, 720, L41

Campbell, S. W., Yong, D., Wylie-de Boer, E. C., et al. 2010, Mem. Soc. Astron. Ital., 81, 1004

Carretta, E., Bragaglia, A., D’Orazi, V., Lucatello, S., \& Gratton, R. G. 2010, A\&A, 519, A71

Carretta, E., Bragaglia, A., Gratton, R., D’Orazi, V., \& Lucatello, S. 2009a, A\&A, 508, 695

Carretta, E., Bragaglia, A., Gratton, R., \& Lucatello, S. 2009b, A\&A, 505,139

Carretta, E., Bragaglia, A., Gratton, R. G., et al. 2006, A\&A, 450, 523

Carretta, E., Bragaglia, A., Gratton, R. G., et al. 2009c, A\&A, 505, 117
Castelli, F., Gratton, R. G., \& Kurucz, R. L. 1997, A\&A, 318, 841

Cavallo, R. M., \& Nagar, N. M. 2000, AJ, 120, 1364

Cohen, J. G., \& Meléndez, J. 2005, AJ, 129, 303

Cudworth, K. M., \& Monet, D. G. 1979, AJ, 84, 774

D'Antona, F., \& Ventura, P. 2007, MNRAS, 379, 1431

D'Ercole, A., D'Antona, F., Carini, R., Vesperini, E., \& Ventura, P. 2012, MNRAS, 423, 1521

D'Ercole, A., D'Antona, F., \& Vesperini, E. 2011, MNRAS, 415, 1304

D'Ercole, A., Vesperini, E., D'Antona, F., McMillan, S. L. W., \& Recchi, S. 2008, MNRAS, 391, 825

Decressin, T., Baumgardt, H., Charbonnel, C., \& Kroupa, P. 2010, A\&A, 516, A73

de Mink, S. E., Pols, O. R., Langer, N., \& Izzard, R. G. 2009, A\&A, 507, L1

Denissenkov, P. A., \& VandenBerg, D. A. 2003, ApJ, 593, 509

Dupree, A. K., Strader, J., \& Smith, G. H. 2011, ApJ, 728, 155

Gratton, R. G., Bonifacio, P., Bragaglia, A., et al. 2001, A\&A, 369, 87

Gratton, R. G., Carretta, E., \& Bragaglia, A. 2012, A\&AR, 20, 50

Gratton, R. G., D’Orazi, V., Bragaglia, A., Carretta, E., \& Lucatello, S. 2010, A\&A, 522, A77

Gratton, R. G., Johnson, C. I., Lucatello, S., D’Orazi D'Orazi, V., \& Pilachowski, C. 2011, A\&A, 534, A72

Gratton, R. G., Sneden, C., \& Carretta, E. 2004, ARA\&A, 42, 385

Iben, I., Jr. 1965, ApJ, 142, 1447

Johnson, C. I., Kraft, R. P., Pilachowski, C. A., et al. 2005, PASP, 117, 1308

Johnson, C. I., \& Pilachowski, C. A. 2010, ApJ, 722, 1373

Kadla, Z. I. 1966, Comm. Pulkova Obs., 24, 93

Kopacki, G., Kołaczkowski, Z., \& Pigulski, A. 2003, A\&A, 398, 541

Kraft, R. P. 1994, PASP, 106, 553

Kraft, R. P., Sneden, C., Langer, G. E., \& Prosser, C. F. 1992, AJ, 104, 645

Kraft, R. P., Sneden, C., Smith, G. H., et al. 1997, AJ, 113, 279

Lardo, C., Bellazzini, M., Pancino, E., et al. 2011, A\&A, 525, A114

Lind, K., Asplund, M., Barklem, P. S., \& Belyaev, A. K. 2011, A\&A, 528, A103

Ludendorf, H. 1905, Publ. Astrophys. Obs. Potsdam, 15

Lupton, R. H., Gunn, J. E., \& Griffin, R. F. 1987, AJ, 93, 1114

Norris, J., Cottrell, P. L., Freeman, K. C., \& Da Costa, G. S. 1981, ApJ, 244, 205

Pasquini, L., Mauas, P., Käufl, H. U., \& Cacciari, C. 2011, A\&A, 531, A35

Peterson, R. C., Rood, R. T., \& Crocker, D. A. 1995, ApJ, 453, 214

Pilachowski, C. A., Sneden, C., Kraft, R. P., \& Langer, G. E. 1996, AJ, 112, 545

Piotto, G. 2009, in Proc. IAU Symposium 258, ed. E. E. Mamajek, D. R. Soderblom, \& R. F. G. Wyse (Cambridge: Cambridge Univ. Press), 233

Prantzos, N., Charbonnel, C., \& Iliadis, C. 2007, A\&A, 470, 179

Sandquist, E. L., Gordon, M., Levine, D., \& Bolte, M. 2010, AJ, 139, 2374

Skrutskie, M. F., Cutri, R. M., Stiening, R., et al. 2006, AJ, 131, 1163

Sneden, C. 1973, ApJ, 184, 839

Sneden, C., Kraft, R. P., Guhathakurta, P., Peterson, R. C., \& Fulbright, J. P. 2004, AJ, 127, 2162

Valcarce, A. A. R., \& Catelan, M. 2011, A\&A, 533, A120

Ventura, P., \& D'Antona, F. 2009, A\&A, 499, 835

Ventura, P., \& D'Antona, F. 2011, MNRAS, 410, 2760 